\section{O risco de parcerias público-privadas em saúde pública pode ser classificado?}

\author{
Can the risk in public-private partnerships \\ be classified?
}

\section{¿Puede ser clasificado el riesgo de las colaboraciones público-privadas en salud pública?}

\section{Resumo}

Durante os próximos anos, Parcerias Público-Privadas (PPP) deverão desempenhar um papel cada vez mais relevante, despontando como uma importante alternativa de financiamento de projetos e de infraestrutura no cenário de serviços públicos. No entanto, especialmente para a Saúde Pública, as PPP nem sempre são uma boa alternativa, uma vez que pode haver distorção da agenda que define as necessidades da saúde, favorecendo os interesses das empresas. Os órgãos públicos podem se beneficiar da colaboração com o setor privado em áreas em que há falta de especialização, tais como desenvolvimento de pesquisas e tecnologias. Mesmo nesses casos, os papéis de cada instituição devem ser bem definidos, para que não haja conflito de interesses. Isso pode ser um desafio quando se trata da formulação de politicas públicas e regulatórias, sobre os impactos que determinadas politicas possam exercer, especialmente sobre nações em desenvolvimento. Envolver-se com o setor privado, sem comprometer a integridade das ações governamentais, exige ampla discussão por parte dos atores da saúde pública, por motivos claros de conflito das visões e escopos entre corporações e saúde pública. Alia-se a isso a necessidade de abordagens multissetoriais, alta carga de investimentos financeiros das várias dimensões das politicas de controle de doenças mais prevalentes, sobretudo as doenças crônicas não transmissíveis (DCNT). Este artigo classifica as PPP em categorias, a fim de minimizar os riscos potenciais de conflito de interesses que podem ter impacto na saúde pública. Essas categorias são definidas como possíveis, possíveis com ressalvas e impossiveis de se envolver com determinadas instituições.

Promoção da Saúde; Parcerias Público-Privadas; Políticas Públicas de Saúde
Vera Luiza da Costa e Silva 1

Silvana Rubano Barretto Turci 1

Ana Paula Natividade de Oliveira 1

Ana Paula Richter 1

doi: 10.1590/0102-311X00086316

\author{
Correspondência \\ A. P. N. Oliveira \\ Escola nacional de Saúde Pública Sergio Arouca, \\ Fundação Oswaldo Cruz. \\ Av. Brasil 4036, sala 909, Rio de Janeiro, Rio de Janeiro, RJ \\ 213040-361, Brasil. \\ anatividade@fiocruz.br \\ 1 Escola Nacional de Saúde Pública Sérgio Arouca, \\ Fundação Oswaldo Cruz, Rio de Janeiro, Brasil.
}




\section{Introdução}

O conceito de ação intersetorial para a saúde é usado, com frequência, para descrever parcerias de diferentes setores envolvidos na promoção da saúde pública, como por exemplo, para dar suporte à implementação e promoção de medidas de prevenção à exposição a fatores de risco, com melhorias na qualidade de vida, por meio de estímulo à realização de atividade física e o desenvolvimento de políticas de nutrição abrangentes, entre outras ações. Essas parcerias podem ser estabelecidas dentro dos governos envolvendo áreas tradicionalmente não relacionadas com a saúde pública, como economia e agricultura. Também podem ocorrer entre segmentos da sociedade, interessados em um determinado tema, como entre a sociedade civil organizada e o setor privado ${ }^{1}$.

As parcerias público-privadas (PPP) vêm despontando num cenário em que as instituições públicas, apesar de apresentarem credibilidade científica, não dispõe de recursos suficientes e emprestam seu nome em troca de patrocínio para a prevenção e tratamento de doenças, assim como para o desenvolvimento de pesquisas, tornando essas parcerias uma alternativa de financiamento de projetos e de infraestrutura ${ }^{2}$.

Embora não haja uma definição consensual do significado de PPP, o Conselho Nacional para Parcerias Público-Privadas americano as define como "um acordo contratual entre um órgão público (federal, estadual ou municipal) e uma entidade do setor privado, em que as habilidades e recursos de cada setor são compartilhados, resultando em um serviço ou em instalações para a uso do público" 3.

A Organização Mundial da Saúde (OMS) descreve as PPP como uma união de um grupo de atores que tem como objetivo comum melhorar a saúde das populações, baseado em papéis e princípios mutuamente acordados ${ }^{4}$. Corroborando essa definição, Reich 5 destaca que as PPP devem ser constituídas por três pontos. Primeiro, devem envolver ao menos uma organização privada com fins lucrativos e uma organização sem fins lucrativos. Segundo, os parceiros devem compartilhar esforços e benefícios. Terceiro, em se tratando de PPP em saúde pública, o objetivo comum dos participantes deve ser a criação de valores sociais para melhorar a saúde de populações em desvantagem.

Ativistas em saúde pública e pesquisadores têm criticado a formação de PPP por entenderem que pode haver distorção da agenda que define as necessidades da saúde pública, favorecendo os interesses das empresas. Tal preocupação tem fundamento porque muitos problemas que deveriam ser resolvidos por ações públicas acabam não sendo priorizados por influência de agentes não governamentais que as modificam de acordo com suas conveniências e interesses 6 .

Não diferente do que ocorre em muitos países, o modelo de desenvolvimento adotado pela grande maioria das empresas que atuam no Brasil se fundamenta em corporativismo e na obtenção de lucro, assim como no investimento de grandes recursos em estratégias de propaganda e marketing para ampliarem sua clientela, além de dar mais credibilidade a seus produtos. Exemplo disso são os brindes oferecidos por indústrias farmacêuticas e de alimentos processados a profissionais de saúde com o objetivo de facilitar essa relação que, muitas vezes, ultrapassa interesses éticos que deveriam ser respeitados 6 .

No Brasil, existem empresas que fomentam parcerias com instituições públicas da área de saúde para promoção de atividades socialmente responsáveis, como combate ao câncer infantil, corrida para a cessação do tabagismo, entre outras. Muitas vezes essas parceiras mostram flagrante contradição. Exemplo disso pode ser visto na participação de empresas na promoção de eventos para reduzir a obesidade e diabetes infantil, mas na verdade produzem alimentos ultraprocessados, calóricos e nutricionalmente pobres, que se constituem em fator de risco para doenças crônicas 7.

A revista Lancet em 2013 publicou os resultados de um estudo do Grupo de Ação para Doenças Crônicas Não Transmissíveis (DCNT), que discute o crescimento da prevalência das DCNT no mundo e o papel das indústrias produtoras de commodities e de produtos insalubres. Essas empresas transnacionais são as principais responsáveis pelo surgimento da epidemia de DCNT e lucram com o aumento do consumo de seus produtos 8. Alguns exemplos clássicos incluem a indústria do tabaco, que tentou fazer acordos voluntários para evitar a regulação do conteúdo de seus produtos, bem como a regulamentação da publicidade e da propaganda, de modo a continuar a promover um produto que mata 1 a cada 2 consumidores e, estima-se, que até 2030 matará mais de 8 milhões de usuários 9 . As indústrias de bebidas alcoólicas, de refrigerantes e de alimentos ultraprocessados vêm utilizando estratégias semelhantes às usadas pela indústria do tabaco para minar políticas custo-efetivas de 
saúde pública. Essas estratégias compreendem não apenas campanhas de publicidade enganosas, uso de embalagens como forma de promover o produto, ausência de informações sobre potenciais riscos do produto ao consumidor, exposição do produto em lugares de destaque e visibilidade voltada para crianças e adolescentes, entre outros 8,10 . Também são demonstrados, de maneira cada vez mais frequente, grupos de organizações que pressionam os governos para impedir a adoção de medidas reguladoras dos produtos, incluindo a ação indireta dos grupos de fachada 11. Nesses casos, deve ser considerado o não estabelecimentos de PPP, tampouco a autorregulação, pois a intervenção do Estado é o único mecanismo que pode evitar/reduzir danos causados por tais indústrias, baseado em evidências científicas. Adicionalmente, pelo mesmo conflito de interesses, essas empresas não devem desempenhar nenhum papel na formulação da política nacional ou internacional para o controle das DCNT 8. O mesmo deve ser dito sobre os grupos de fachada dessas empresas, que não devem ser aceitos como parceiros.

Observando o histórico recente de leis restritivas com o objetivo de reduzir o consumo de fatores de risco, indústrias multinacionais como a de tabaco, bebidas alcóolicas, de refrigerantes e de alimentos ultraprocessados têm tentado influenciar governos para firmar acordos voluntários com a alegação de não sofrerem queda brusca no seu faturamento 12,13 .

A indústria de tabaco não raro tem promovido eventos sobre cidadania, participado de fóruns que discutem o papel das empresas na manutenção de reservas naturais, e é membro do Pacto Global (UN Global Compact) das Organizações das Nações Unidas que prevê ações responsáveis sobre o meio ambiente, direitos humanos e trabalhistas ${ }^{14}$. Contradição semelhante ocorre com a indústria de bebidas alcóolicas, ao patrocinar eventos esportivos e promover campanhas publicitárias direcionadas aos jovens, pois sabidamente seu produto está associado ao desenvolvimento das principais DCNT, como diabetes, hipertensão arterial e câncer; e suas estratégias de marketing funcionam mais no sentido de aumentar o uso do álcool socialmente e aumentar a prevalência de doenças 15,16 . A Convenção-Quadro para o Controle do Tabaco da Organização Mundial da Saúde (CQCT-OMS) traz, entre seus instrumentos, um artigo específico e guias para sua implementação, objetivando lidar com a interferência da indústria do tabaco (Artigo 5.3) e encorajar os governos a proteger as políticas públicas de controle do tabaco dessas interferências 17.

No entanto, existem PPP desejáveis e viáveis. Os órgãos públicos podem se beneficiar da colaboração com o setor privado em áreas em que há falta de especialização, tais como desenvolvimento de pesquisas e tecnologias. Mas, mesmo nesses casos, os papéis que serão executados por cada instituição devem ser bem definidos antes de qualquer passo concreto, para que não haja conflito de interesses. Isso pode ser um desafio quando se trata da formulação de políticas públicas e regulatórias, particularmente sobre os impactos que determinadas políticas possam exercer sobre nações em desenvolvimento 18 .

Este artigo discute quais parcerias público-privadas podem ser estabelecidas na área da saúde pública e como os governos dos países devem agir para se proteger quando houver conflito de interesses.

\section{Metodologia}

Este artigo procura refletir as diferentes estratégias usadas na formação das PPP. Para tanto, foi feita uma revisão narrativa de literatura, consultando livros, periódicos e literatura cinzenta, selecionados nas bases de dados LILACS, SciELO, PubMed e Periódico Capes, para artigos científicos e Internet para a literatura cinzenta. Os descritores e os respectivos termos usados nas buscas foram: parcerias público-privadas, parcerias AND saúde, parcerias AND promoção da saúde, parcerias AND prevenção de doenças crônicas não transmissíveis, políticas de saúde. Foram considerados para analise os documentos publicados no período de 1998 até 2015, tanto em inglês quanto em português. A pesquisa foi realizada no período de agosto de 2013 a abril de 2016. 


\section{Discussão}

Para Kraak et al. ${ }^{19}$, seis desafios devem ser considerados nas PPP com o objetivo de diminuir os riscos decorrentes dessas parcerias na área da saúde pública: (i) equilíbrio dos interesses privados com os interesses da saúde pública; (ii) gestão de conflitos de interesses e preconceitos; (iii) assegurar que associações na utilização de marcas apoiem produtos e ambientes saudáveis; (iv) cumprimento de códigos éticos de conduta; (v) realização de dupla diligência para avaliar a compatibilidade da parceria; e (vi) acompanhamento e avaliação dos resultados da parceria.

Países desenvolvidos como o Reino Unido, declaram ter conseguido pactuar metas que têm impacto sobre a saúde da população como a parceria que fizeram com empresas privadas do setor de alimentos e a redução nos teores de sal, o que consequentemente traz benefícios imediatos sobre a pressão arterial e sobre o desenvolvimento de DCNT. A sociedade civil desempenhou um papel importante nesta proposta, pois é ela quem monitora e fiscaliza o cumprimento desses acordos 20,21.

De fato, as PPP na área da saúde devem ser minuciosamente avaliadas e, com tal objetivo a Organização das Nações Unidas (ONU) criou o Comitê permanente que define regras para o engajamento com o setor privado. Esse comitê elabora manuais para orientar a interação, por exemplo, entre o governo e empresas de alimentos, observando que seja mantido o diálogo aberto e claro sobre potenciais conflitos de interesse e garantindo que esses aspectos sejam adequadamente gerenciados 22 .

Outro bom exemplo são as orientações sobre estratégias que estão sendo adotadas para conter o consumo de bebidas alcóolicas. Essas diretrizes propõem a regulação do conteúdo, do volume de comercialização, seja ela feita mediante marketing direto ou indireto, em algumas ou todas as mídias; das atividades de patrocínio; sobre medidas restritivas de acesso ou proibição quando os produtos são destinados aos jovens 23 .

\section{Classificando parcerias público-privadas}

O uso das PPP em saúde pública exige soluções inovadoras. Exemplos podem ser encontrados em diversos países e, desde que respeitadas as regras estabelecidas pelos órgãos reguladores dos governos, as PPP podem oferecer meios adicionais para a pesquisa em saúde pública. No entanto, o saldo entre perdas e ganhos pode não estar claro 24 . De acordo com a análise dos autores, as PPP poderiam ser divididas em possíveis, possíveis com ressalvas e impossíveis.

\section{Parcerias possíveis}

Um exemplo de sucesso desse tipo de parceria é o Transport, Health and Environment Pan-European Programme (THE PEP), coordenado pelo Escritório Regional da OMS para a Europa, em que foram desenvolvidas orientações e ferramentas práticas sobre os efeitos benéficos para a saúde e economia adquiridos com a prática do ciclismo e caminhada. As peças de comunicação foram desenvolvidas com revisão sistemática de pesquisas relevantes que envolveram especialistas com ampla experiência e conhecimento nas áreas de saúde pública, epidemiologia, transportes e economia. As ferramentas geradas pelo projeto eram fáceis de usar e foram adotadas com sucesso por países de alta, média e baixa renda 25,26. Esses projetos mostram que o uso de argumentos econômicos para defender investimentos em políticas sociais pode eventualmente trazer claros benefícios para a saúde 27 .

Outro projeto de sucesso envolvendo PPP foi o North Karelia Project, desenvolvido pelo governo da Finlândia nos anos 60, com o objetivo de reduzir as elevadas taxas de doenças cardiovasculares presente na população. Foram propostas mudanças no estilo de vida e de consumo da população, com a colaboração da sociedade civil e do setor privado aos setores regulatórios do governo, e também acordos com algumas indústrias, especialmente de alimentos. As taxas de DCNT caíram vertiginosamente e, até hoje, essa PPP é considerada um modelo que deve ser seguido por todos os países 28 .

O Departamento de Saúde do Reino Unido, em parceria com a Agência de Normas para Alimentos do Reino Unido, considerou as recomendações do Comitê Científico Consultivo em Nutrição do Reino Unido (SACN) de que o consumo médio de sal da população deveria ser reduzido porque os níveis de ingestão eram, em média, da ordem de $9 \mathrm{~g} /$ dia, e deveriam ser no máximo $6 \mathrm{~g} / \mathrm{dia}$ para os adultos e ainda menor para as crianças. Para que houvesse o efetivo cumprimento dessa recomendação, as auto- 
ridades do Reino Unido tiveram que trabalhar com vários setores da indústria de alimentos, incluindo os varejistas, fabricantes, associações comerciais, fornecedores da indústria, academia, organizações de voluntários e autoridades locais 20 .

\section{Parcerias possíveis com ressalvas}

As PPP podem se traduzir em avanços para a saúde da população no presente, enquanto a criação de fluxos de inovação pode gerar futuros dividendos sociais e financeiros para reforçar a saúde pública de forma mais ampla. É necessária uma avaliação por parte da academia para que haja uma discussão científica fecunda e que contribua para um refinamento das PPP e gere diretrizes para a saúde pública 29.

Como o lucro é sempre o principal objetivo de uma grande corporação, há sempre que se pensar no foco da saúde pública antes de uma parceria entre saúde e uma indústria. Desde que o objetivo principal da parceria seja diminuir níveis de substâncias danosas à saúde.

No entanto, torna-se mais complexo firmar uma parceria com empresas que sabidamente comercializam produtos nocivos à saúde, como no caso das indústrias de refrigerantes, de alimentos processados (fast foods), biscoitos, e essas parcerias não tenham as salvaguardas de uma melhoria ou redução de substâncias nocivas. Para que haja um real ganho para a saúde pública, com melhor controle das DCNT, é preciso estabelecer compromissos de redução ou mesmo erradicação de substâncias prejudiciais à saúde humana e ao meio ambiente ${ }^{7}$.

Dentre essas parcerias com salvaguardas, estão as indústrias dos alimentos processados, bebidas não alcoólicas (em especial os refrigerantes) e a indústria farmacêutica. Uma vez que os interesses industriais e corporativos não se sobreponham aos interesses da saúde pública, tais parcerias podem ser viáveis 19 .

Com relação à indústria farmacêutica, é necessário avaliar se a parceria ou patrocínio de pesquisas em saúde não influenciam nos resultados, favorecendo a prescrição de medicamentos. Para Thompson 30, deve se considerar um conjunto de condições e circunstâncias impostas por indústrias e instituições comerciais (conflito de interesses) que podem influenciar negativamente na conduta do profissional de saúde em relação ao interesse primário (que deveria ser o bem-estar e tratamento do paciente) e a validade de pesquisas investigativas. Isso pode ser prejudicado pelo interesse secundário, como proveito econômico, desejo de notoriedade, prestígio e reconhecimento profissional. Outro exemplo de parceria com ressalvas é o Special Programme for Research and Training in Tropical Diseases (TDR) da OMS, financiado pelo Banco Mundial, o Fundo das Nações Unidas para a Infância (UNICEF), o Programa de Desenvolvimento das Nações Unidas (PNUD) e a OMS. Ests programa tem por objetivo facilitar, apoiar e influenciar a colaboração científica em prol do combate das doenças tropicais negligenciadas e relacionadas com a pobreza, como malária, leishmaniose, dengue e tuberculose 31 . Apesar de ser uma iniciativa do setor público, o TDR conta com a participação da indústria farmacêutica, e tal parceira foi e continua a ser cautelosamente monitorada pelo Joint Coordinating Board (JCB) do TDR. Dessa maneira, o JCB garante os interesses superiores protetivos da saúde, por intermédio do mútuo respeito entre as instituições participantes, de objetivos claramente definidos, da transparência nas prestações de conta para as agências financiadoras, assim como para o público em geral e dos direitos do setor público de propriedade intelectual que são produzidos pelos esforços conjuntos com a indústria 32 .

\section{Parcerias impossíveis}

Em parcerias público-privadas, como em qualquer parceria, a necessidade de transparência e interesse mútuo é imprescindível. Assim, parcerias público-privadas com a indústria fumageira, indústria armamentista e indústria de bebida alcoólica são impossíveis de serem realizadas, tendo em vista a impraticabilidade de haver um interesse mútuo entre os dois setores. Ademais, todas as tentativas realizadas para o estabelecimento de acordos voluntários com algumas dessas indústrias não tiveram nenhum resultado positivo para a sociedade, pelo contrário, retardaram a implementação de medidas que pudessem etiquetar e reduzir sua atratividade e consumo ${ }^{8}$.

O comércio ilícito de tabaco é um problema internacional e o seu combate é um componente indispensável para o controle do tabagismo, uma vez que o comércio ilícito mina os objetivos de 
saúde dos países ao enfraquecer suas políticas de aumento de preços e impostos. Um exemplo de parceria impossível é a firmada entre a International Criminal Police Organization (Interpol) e as quatro indústrias transnacionais do tabaco (British American Tobacco, Imperial Tobacco Group, Japan Tobacco International e Philip Morris International) por força da qual a Interpol utiliza o sistema de rastreio e localização de produtos do tabaco, chamado Codentify, desenvolvido pela Philip Morris International. Essa parceria entre a indústria do tabaco e uma autoridade internacional, como a Interpol, não é adequada para quem atua na defesa de práticas ilícitas que afetam a saúde, já que ela inclui a indústria do tabaco como parte no combate ao comércio ilícito, o que permite e favorece a influência dela nas políticas de controle do tabagismo e vai de encontro à implementação do Artigo 5.3 da CQCT-OMS e o Artigo 8 do Protocolo de Comércio Ilícito da CQCT-OMS 33.

Uma síntese das parcerias possíveis, possíveis com ressalvas e impossíveis é proposta na Tabela 1.

\section{Considerações finais}

Não é raro identificar empresas que se comportam como "lobos em pele de cordeiro" e realizam campanhas publicitárias milionárias para promover seus produtos nocivos à saúde, associando esses produtos à promoção de bem-estar, alegria e sucesso.

Como lidar com empresas cujo principal objetivo é o lucro? Como estabelecer uma relação "promissora e saudável” entre indústrias e profissionais de saúde em que muitas vezes existem interesses opostos? Como lidar com as empresas quando há nítido conflito de interesses? A resposta para tais perguntas é unicamente priorizar o interesse da saúde pública em proteger a população de consumir produtos nocivos à saúde, seja na regulação da publicidade, seja na redução de teores de substâncias nocivas ou na oferta de alimentos mais saudáveis.

É importante considerar a análise feita pela OMS 18 sobre quais princípios devem ser adotados a fim de tornar as PPP bem-sucedidas, lembrando que devem ser aplicados, independentemente da natureza da causa e do contexto, seja ela nacional ou internacional:

a) no caso da OMS que é composta por Estados-Membros e por um Secretariado, a tomada de decisões cabe exclusivamente aos órgãos responsáveis pela organização política do acordo, ou seja, nenhum ator não estatal pode esperar obter privilégios na mesma base que os Estados-Membros. A OMS é uma organização científica e faz suas abordagens em saúde pública com base em evidências científicas;

b) o desenvolvimento de normas, padrões, decisões políticas e as estratégias devem continuar a ser baseados no uso sistemático de provas e devem ser protegidos da influência de qualquer maneira de preconceito velado, interesse comercial ou de qualquer outra forma de influência indevida;

Tabela 1

Tipos de Parceiras Público-Privadas de acordo com setores da economia e viabilidade.

\begin{tabular}{|c|c|c|c|}
\hline Tipo de parcerias & Tipos de empresa & Motivação & Viabilidade \\
\hline Possíveis & $\begin{array}{c}\text { Esporte } \\
\text { Alimentos não processados }\end{array}$ & $\begin{array}{l}\text { Promove saúde com seu produto ou seu } \\
\text { consumo não causa danos. }\end{array}$ & $\begin{array}{c}\text { Firmar parcerias para pesquisa e } \\
\text { desenvolvimento. }\end{array}$ \\
\hline Possíveis com ressalvas & $\begin{array}{l}\text { Alimento } \\
\text { Indústria farmacêutica } \\
\text { Alimento processado }\end{array}$ & $\begin{array}{l}\text { Produto provoca doenças, como DCNT, se } \\
\text { consumido inadvertidamente. }\end{array}$ & $\begin{array}{l}\text { Fazer parcerias com salvaguardas } \\
\text { sujeitas a regulação de publicidade, } \\
\text { promoção e patrocínio. } \\
\text { Compromisso de redução de teores } \\
\text { de produtos nocivos. }\end{array}$ \\
\hline Impossíveis & $\begin{array}{l}\text { Indústria fumageira } \\
\text { Indústria de bebidas alcoólicas } \\
\text { Indústria armamentista }\end{array}$ & $\begin{array}{l}\text { Produto reconhecidamente danoso à } \\
\text { saúde, podendo levar à morte. }\end{array}$ & $\begin{array}{c}\text { Jamais fazer parcerias entre setores } \\
\text { públicos e estas indústrias. }\end{array}$ \\
\hline
\end{tabular}

DCNT: doenças crônicas não transmissíveis. 
c) transparência é o que salvaguarda e sustenta as relações público-privadas. Esse princípio implica que a OMS torne pública a natureza de suas relações com a iniciativa privada e, por sua vez, as empresas privadas que tiveram qualquer relação com a OMS são obrigadas a tornarem públicos seus objetivos organizacionais, suas estruturas institucionais, suas fontes de financiamento e a natureza de seu relacionamento com a OMS;

d) a declaração de "Conflitos de Interesses", seja ele real ou percebido, individual ou institucional, deve ser adequadamente gerenciada e explicitada, de modo que todos os interessados estejam cientes deles.

PPP serão exitosas somente quando houver um marco regulatório que priorize a saúde pública. Os governos e autoridades da saúde, com conhecimento científico disponível sobre os fatores de riscos para desenvolvimento de doenças, têm a obrigatoriedade de determinar o que é permitido, e as empresas devem adequar-se à regulação. Desse modo, as parcerias podem ser profícuas.

\section{Colaboradores}

V. L. Costa e Silva, S. R. B. Turci, A. P. N. Oliveira e A. P. Richter participaram da redação e revisão crítica do conteúdo intelectual do manuscrito e aprovação final da versão a ser publicada.

\section{Referências}

1. Buss PM. Promoção da saúde e qualidade de vida. Ciênc Saúde Coletiva 2000; 5:163-77.

2. Hardcastle C, Boothroyd K. Risk overview in public-private partnership. In: Akintola A, Beck M, Hardcastle C, editors. Public-private partnerships: managing risks and opportunities. Oxford: Blackwell Science; 2008. p. 31-57.

3. The National Council for Public-Private Partnership. Overvew \& mission. http://www. ncppp.org/ (acessado em 09/Jan/2014).

4. Kickbusch I, Quick JD. Partnerships for health in the 21st century. World Health Stat Q 1998; 51:68-74.

5. Reich MR. Public-private partnerships for public health. Nat Med 2000; 6:617-20.

6. Departamento de Ações Programáticas Estratégicas, Secretaria de Atenção à Saúde, Ministério da Saúde. A legislação e o marketing de produtos que interferem na amamentação: um guia para o profissional de saúde. Brasília: Ministério da Saúde; 2009. (Série A. Normas e Manuais Técnicos)

7. Freedhoff Y, Hébert PC. Partnerships between health organizations and the food industry risk derailing public health nutrition. CMAJ 2011; 183:291-2.

8. Moodie R, Stuckler D, Monteiro C, Sheron N, Neal B, Thamarangsi T, et al. Profits and pandemics: prevention of harmful effects of tobacco, alcohol, and ultra-processed food and drink industries. Lancet 2013; 381:670-9.

9. World Health Organization. Report on the global tobacco epidemic 2013: Enforcing bans on tobacco advertising, promotion and sponsorship. Geneva: World Health Organization; 2013. 
10. da Costa e Silva VL, Pantani D, Andreis M Sparks R \& Pinsky I. Bridging the gap between science and public health: taking advantage of tobacco control experience in Brazil to inform policies to counter risk factors for non-communicable diseases. Addiction 2013; 108:1360-6.

11. World Health Organization. WHO report on the global tobacco epidemic, 2015: raising taxes on tobacco. Geneva: WHO Press; 2015.

12. Cavalcante TM. O controle do tabaco no Brasil: avanços e desafios. Rev Psiquiatr Clín (São Paulo) 2005; 32:283-300.

13. Malta DC, Silva Junior, JB. Brazilian strategic action plan to combat chronic non-communicable Diseases and the global targets set to confront these diseases by 2025: a review. Epidemiol Serv Saúde 2013; 22:151-64.

14. United Nations Global Compact. Our participants. https://www.unglobalcompact. org/what-is-gc/participants (acessado em Abr/2016).

15. Duncan BB, Chor D, Aquino EML, Bensenor IM, Mill JG, Schimidt MI, et al. Doenças crônicas não transmissíveis no Brasil: prioridade para enfrentamento e investigação. Rev Saúde Pública 2012; 46:126-34.

16. Leppo K, Ollila E, Peña S, Wismar M, Cook $\mathrm{S}$. Health in all policies: seizing opportunities, implementing policies. Helsinki: Ministry of Social Affairs and Health; 2013.

17. World Health Organization. WHO Framework Convention on Tobacco Control. http:// apps.who.int/iris/bitstream/10665/42811/ 1/9241591013.pdf (acessado em Abr/2016).

18. World Health Organization. Trade, foreign policy, diplomacy and health: public-private partnerships for health. http://www.who.int/ trade/glossary/story077/en/ (acessado em Abr/2016).

19. Kraak VI, Harrigan PB, Lawrence M, Harrison PJ, Jackson MA, Swinburn B. Balancing the benefits and risks of public-private partnerships to address the global double burden of malnutrition. Public Health Nutr 2012; 15:503-17.

20. UK Food Standards Agency. Salt reduction initiatives. http://www.food.gov.uk/multimedia/ pdfs/saltreductioninitiatives.pdf (acessado em Abr/2016).

21. World Economic Forum. Public-private partnerships in health. The private sector's role in Public-Private Partnerships. https://www.we forum.org/ (acessado em Abr/2016).
22. United Nations System Standing Committee on Nutrition. SCN private sector engagement policy. http://www.unscn.org/en/home/ (acessado em Abr/2016).

23. United Nations System Standing Committee on Nutrition. Nutrition and business: how to engage? Geneva: United Nations System/ World Health Organization; 2011.

24. Reijneveld SA. Public-private partnerships in public health research: does it work and if yes, how? Eur J Public Health 2012; 22:165-6.

25. United Nations Economic Commission for Europe. Transport, Health, and Environment Pan-European Programme (THE PEP). http:// www.unece.org/thepep/en/welcome.html (acessado em Abr/2016).

26. WHO Regional Office for Europe. Health economic assessment tools (HEAT) for walking and for cycling: methodology and user guide. Geneva: WHO Regional Office for Europe; 2011.

27. Kohl HW 3rd, Craig CL, Lambert EV, Inoue $\mathrm{S}$, Alkandari JR, Leetongin G, et al. The pandemic of physical inactivity: global action for public health. Lancet 2012; 380:294-305.

28. Puska P, Vartiainem E, Laatikainem T, Jousilahti P, Paavola M. The North Karelia Project: from North Karelia to national action. Helsink: Helsinki University Printing; 2009.

29. De Pinho Campos K, Norman CD, Jadad AR. Product development public-private partnerships for public health: a systematic review using qualitative data. Soc Sci Med 2011; 73: 986-94.

30. Thompson DF. Understanding financial conflicts of interest. N Engl J Med 1993; 329:573-6.

31. World Health Organization. Special Programme for Research and Training in Tropical Diseases (TDR). http://www.who.int/tdr/en/ (acessado em Abr/2016).

32. Reich MR. Public-private partnerships for public health. Cambridge: Harvard University Press; 2002.

33. Joossens L, Gilmore AB. The transnational tobacco companies' strategy to promote $\mathrm{Co}$ dentify, their inadequate tracking and tracing standard. Tob Control 2014; 23:e3-6. 


\section{Abstract}

In the coming years, public-private partnerships (PPPs) should play an increasingly relevant role as an important alternative for financing projects and infrastructure in public services. However, especially in public health, PPPs are not always a good alternative, since they may introduce distortions in the agenda that sets health needs, favoring companies' interests. Public agencies can benefit from collaboration with the private sector in areas where there is a lack of specialization, such as the development of research and technologies. Even in these cases, each institution's role needs to be defined in order to avoid conflicts of interest. This can be challenging when dealing with the formulation of public and regulatory policies, on the impacts of certain policies, especially in developing countries. To engage with the private sector without compromising the integrity of government actions requires a broad discussion by public health stakeholders, for clear reasons of conflicting visions and scopes between corporations and public health. Combined with this is the need for multisector approaches, with a high load of financial investments in the various dimensions of policies to control the most prevalent diseases, especially chronic non-communicable diseases (NCD). This article classifies PPPs in categories in order to minimize the potential risks of conflicts of interest than can impact public health. These categories are defined as possible, possible with caveats, and impossible for involvement with certain institutions.

Health Promotion; Public-Private Sector Partnerships; Public Health Policy

\section{Resumen}

Durante los próximos años, las colaboraciones público-privadas (PPP) por sus siglas en portugués) deberán desempeñar un papel cada vez más relevante, despuntando como una importante alternativa de financiación de proyectos y de infraestructura en el escenario de servicios públicos. No obstante, especialmente para la Salud Pública, las PPP no siempre son una buena alternativa, ya que puede haber distorsiones de la agenda que define las necesidades de la salud, favoreciendo los intereses de las empresas. Los órganos públicos pueden beneficiarse de la colaboración con el sector privado en áreas donde hace falta especialización, tales como: desarrollo de investigaciones y tecnologías. Incluso en esos casos, los papeles de cada institución deben ser bien definidos para que no exista un conflicto de intereses. Eso puede ser un desafío cuando se trata de la formulación de politicas públicas y regulatorias, sobre los impactos que determinadas politicas puedan ejercer, especialmente sobre naciones en desarrollo. Involucrarse con o el sector privado, sin comprometer la integridad de las acciones gubernamentales, exige una amplia discusión por parte de los actores de la salud pública, por motivos claros de conflicto de las visiones y objetivos entre corporaciones y salud pública. Se une a esto la necesidad de enfoques multisectoriales, alta carga de inversión financiera de las diferentes dimensiones de las politicas de control de enfermedades más prevalentes, sobre todo las enfermedades crónicas no transmisibles (DCNT). Este artículo clasifica las PPP en categorías, a fin de minimizar los riesgos potenciales de conflicto de intereses que pueden tener un impacto en la salud pública. Estas categorías se definen como posibles, posibles con salvaguardias e imposibles de implicación con determinadas instituciones.

Promoción de la Salud; Asociaciones entre el Sector Público y el Privado; Políticas Públicas de Salud 